\title{
Upcoming Events Related to Electrocatalysis
}

Published online: 20 July 2013

(C) Springer Science+Business Media New York 2013

\section{October 2013}

224th Meeting (Fall Meeting), The Electrochemical Society, 27 October-1 November 2013, San Francisco, CA, USA Event website: http://www.electrochem.org/meetings/biannual/ $224 /$

\section{November 2013}

Surface Modification for Chemical and Biochemical Sensing, 8-12 November 2013, Łochów, Poland

Event website: www.smcbs2013.pl

24th Annual Conference of the Catalysis Society of South Africa, Electrocatalysis Session, 17-20 November 2013, Wild Coast Sun near Port Edward, South Africa Event website: http:/www.catsa2013.ukzn.ac.za/

\section{December 2013}

Sao Paulo School of Advanced Sciences on Electrochemistry, Energy Conversion and Storage (8th School of Electrochemistry), 7-14 December 2013, São Paulo, Brazil Event website: www.usp.br/escoladeeletroquimica

February 2014

ISEAC Discussion Meet on Electrochemistry and its Applications, 20-25 February 2014, Amritsar (Punjab), India Event website: www.iseac.org 